\title{
UNIDIRECTIONAL RADIOBEACON FOR AIRCRAFT
}

\author{
By E. Z. Stowell
}

\begin{abstract}
A method of transmitting unidirectional beacon signals for the aural or visual guidance of aircraft has been worked out. Such signals increase the efficiency of the beacon from the power standpoint, reduce interference from other beacons, and reduce the number of radiated courses to one. It is believed that the polar characteristic of the radiated field is about the optimum for aircraft use.

The method consists in transmitting directive and nondirective fields simultaneously, with the proper phase and amplitude relations between them to secure unidirectional transmission.

\section{CONTENTS}

I. Introduction

II. Principle of the directive beacon

III. Determination of field shape _.

IV. Production of a unidirectional field _.

V. Summary _._.
\end{abstract}

\section{INTRODUCTION}

During the development by the Bureau of Standards of a directive beacon for guiding aircraft ${ }^{1}$ it appeared that the usefulness of the beacon could be increased by making the radiated field unidirectional. As the operation of a considerable number of beacons was contemplated, all on the same frequency of $290 \mathrm{kc}(1,034 \mathrm{~m})$, a large amount of the interference between them might be prevented in this way. In addition, more signal intensity might be obtained in a desired direction with a given amount of power, and the pilot would be less subject to confusion from a multiplicity of possible courses.

These considerations had sufficient weight to warrant experimentation. The attempts to obtain such a unidirectional arrangement without seriously departing from the directive beacon circuits as they then stood are described in this paper.

\section{PRINCIPLE OF THE DIRECTIVE BEACON}

The beacon system, without the unidirectional feature, has been fully described. ${ }^{1}$ The principles involved will be briefly stated here.

\footnotetext{
1 Development of radio aids to air navigation, J. H. Dellinger and H. Pratt, Proc. I. R. E., 16, pp. 890-920, July, 1928.
}

$10488^{\circ}-28$ 
Figure 1 shows a schematic diagram of the arrangement. A master oscillator supplies power at $290 \mathrm{kc}$ to two power-amplifier tubes. These operate alternately, say with a 500-cycle plate supply. In the plate circuits are the stator coils of a goniometer, the secondaries of which are in series with their respective coil antennas at right angles.

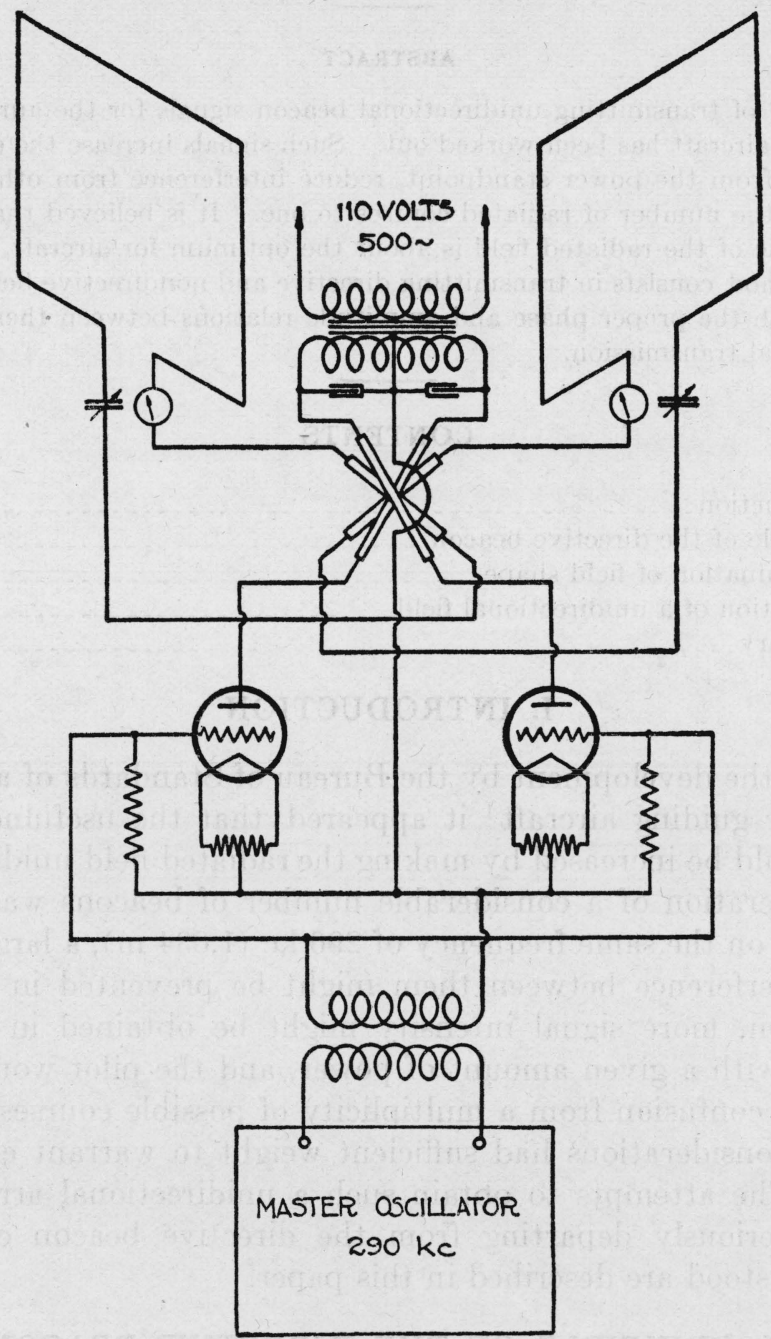

Fig. 1.-Principle of beacon transmitter

It can be demonstrated that the resultant signal is that emitted by two radiating loops (coil antennas), the size of the actual loops used, but inclined to each other by the angle between the stator coils of the goniometer, and whose apparent orientation can be shifted by rotating the goniometer coils (either pair). These equivalent loops are some- 
times called the "phantom loops" to distinguish them from the physical loops carrying the power to be radiated.

Given a fundamental circuit arrangement of this kind, a number of ways can be found to make the modulation mark out lines in space which radiate from the transmitter like the spokes of a wheel. For example, in the above system there will be four directions from the transmitter in which a 1,000-cycle note will be emitted. These are the directions in which the signals from the two phantom loops are received with equal intensity. These directions are theoretically lines, or zones of no width, but they actually have a small width depending on the discrimination of the receiving operator or receiving equipment. Hence, these directions are generally referred to as equisignal zones.

In all other directions the received note will be 500 cycles. Since these four directions $90^{\circ}$ apart can be swung around in space together, we have a flexible system for indicating a desired direction to an airplane. In practice such a system would be useless, for it would be quite difficult for an airplane which accidentally wandered off - this course to find it again. It turns out that an additional feature must be provided which informs the airplane which side of the course is right and which is left. This feature does not concern the present paper, and it is only necessary to state that if the above fundamental system can be gotten to radiate a unidirectional field, the modifications necessary to insure practical working with an airplane follow naturally. Accordingly, most of the experimental work was done with the above arrangement.

\section{DETERMINATION OF FIELD SHAPE}

A single loop radiates a figure of eight because of the phase interference from the parallel sides. Two loops sending alternately would, therefore, radiate two figures of eight inclined to one another by the angle between the loops. In the case of the beacon transmitter of Section II, if the primary coils of the goniometer are placed at $60^{\circ}$, the emitted field is that of two loops at $60^{\circ}$, shown in Figure $2(a)$. If the coils are at right angles, the field is that of Figure $2(b)$.

In case the alternations from one loop to another are sufficiently rapid, the detecting device can not follow them, and the two fields overlap to give the resultant fields in these figures. Such is the case, for example, with 500-cycle plate supply at the transmitter and a thermoelement at the receiving end. Since most reception devices would integrate the signal in this manner, the measurements of field shape were made upon the "resultant fields," generally with a thermoelement at the output of a receiving set, sometimes with a 2-electrode detector feeding a Leeds \& Northrup automatic recorder. 



\section{PRODUCTION OF A UNIDIRECTIONAL FIELD}

Methods of transmitting unidirectional signals fall into two broad classes. One employs the antenna array and is probably the most widely used. It consists of a number of antennas appropriately spaced and excited at the proper times from the same source. Quite varied fields have been gotten in this manner, which have been discussed in the literature. ${ }^{2}$ These methods are open to two objections for the purpose of the present beacon-the amount of land necessary might be excessive and the design is radically different from the beacon as already developed. However, a beacon operating in one of the high-frequency bands might be economically constructed on this principle.

The second group of methods approaches more nearly the needs of the present beacon. This group is characterized by the superposition of a nondirectional field upon a bilateral field (such as a figure of eight) to produce a unilateral field (such as a cardioid). This was first done by Bellini and Tosi. ${ }^{3}$ They employed two triangular loops at right angles, open at the top, and excited from a goniometer. A single vertical wire was supplied with power from an extra winding on the goniometer that supplied the loops. By adjusting phase and amplitude relations between the directive and nondirective fields a cardioid could be radiated in the most favorable case. This was proved by reception measurements. The cardioid could be oriented in any direction by rotating the goniometer.

With this as a clue, experiments were made upon the beacon at College Park, Md., with a vertical wire 70 feet long, radiating simultaneously with the loops. Various methods of feeding were tried, the circuit arrangement of Figure 3 giving best results. Figure 4 shows the shape of the field as received on an automatic recorder at the Bureau of Standards, 11 miles distant, while the goniometer was rotated at a constant rate. The dip at $270^{\circ}$ shows the effect of the vertical wire. The effect is not very pronounced, due to lack of power in the wire. The wire was tuned by loading it with a variometer; hence, the currents in the wire and the loops are in phase or nearly so.

In order to increase the effect of the nondirective field, it became necessary to use a more efficient radiator. Such a radiator was already at hand in the loop structure. (See fig. 5.) The connection

${ }^{2}$ F. Braun, Electrician, 57, pp. 222 et seq., 244 et seq.; 1906 . Bellini, Electrician, 74 , p. 352; $1914 . \quad$ R. M. Foster, Bell System Tech. J., 5, p. 292; April, 1926. Blondel, Compt. Rend., 184, pp. 561, 721 ; 1927. Richtcharakteristiken von Antennenkombinationen, by A. Esau, Jahrb. d. drahtl. Tel. und Tel., 27, pp. 28, 142; 1926 (in 3 parts). Directive transmission with grid antennas, Mesny, L'Onde Electrique, 6, p. $181 ; 1927$. Approximate theory of the flat projector (Franklin) aerial used in the Marconi beam system of wireless telegraphy, J. A. Fleming, Exp. Wireless, 4, p. 287; 1927.

${ }^{3}$ Bellini and Tosi, A directive system of wireless telegraphy, Electrician, pp. 62, 531; 1909 . Murphy and Wolfe, Stationary and rotating equisignal beacons, J. Soc. Auto. Engrs.; September, 1926. 


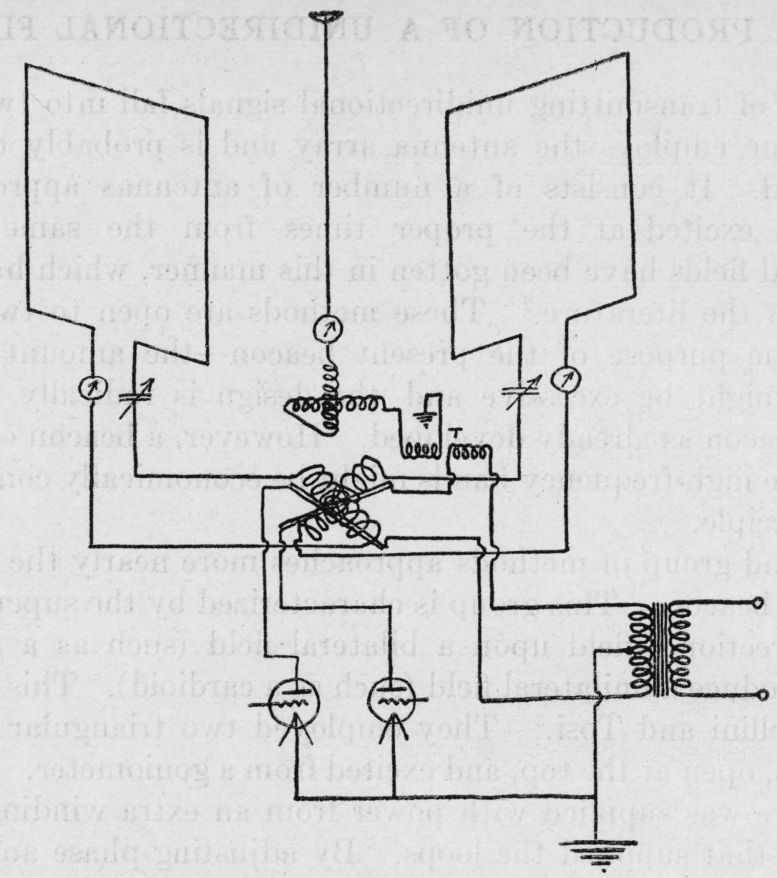

FIG. 3.-Excitation of vertical wire from goniometer circuit

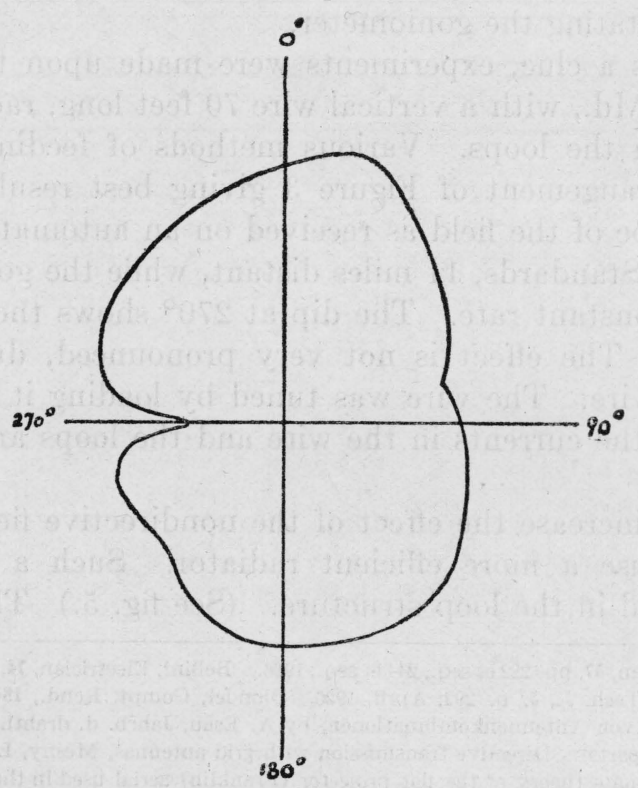

FIG. 4.-Field of loops and vertical wire 


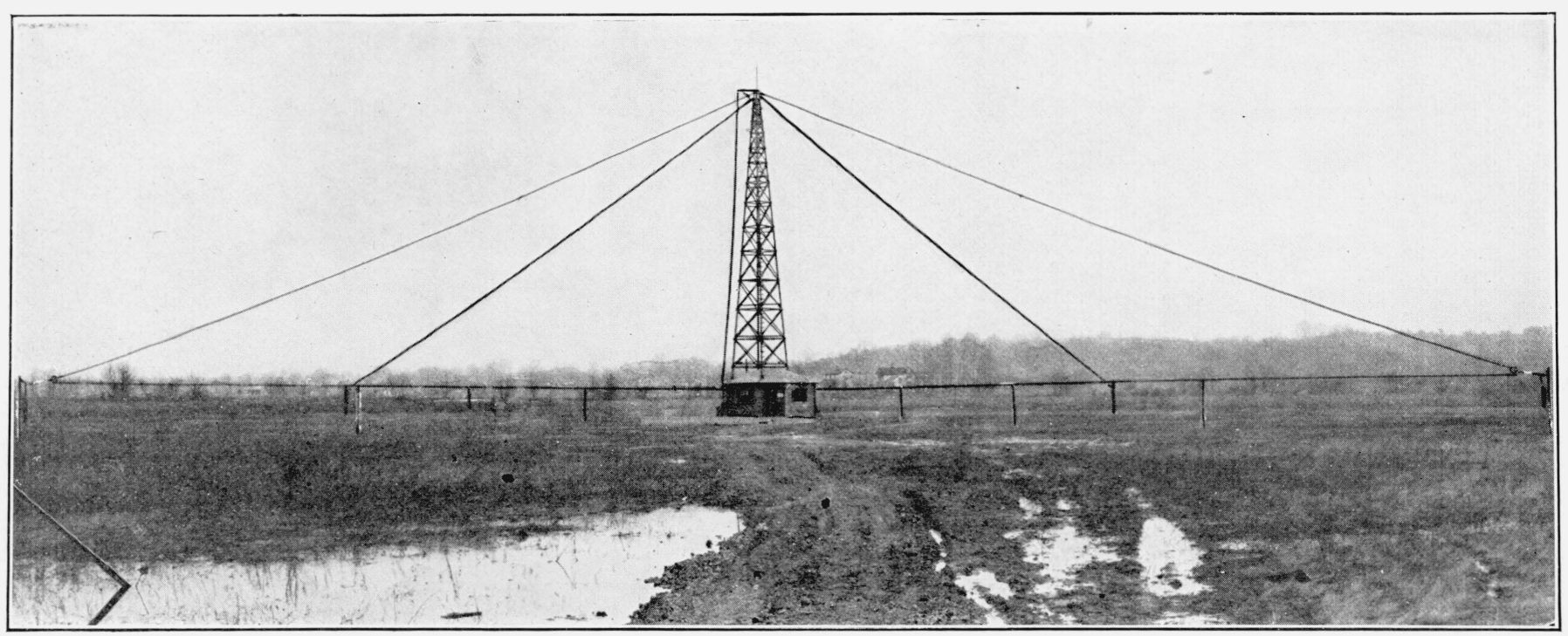

FIG. 5.-Antennas of experimental beacon at College Park, Md. 
to the vertical wire was replaced by a connection to the common mid-point of the loops as in Figure 6 .

This connection was without effect on the circulating currents in the loop themselves; that is, the loops could be connected together at their mid-points and grounded without changing the readings of the ammeters in series with the loops. The increased effectiveness is shown by the fact that in Figure 6 the ammeter in series with the variometer registered 3 to 5 amperes with the power available, as against 1 ampere with the connection of Figure 3.

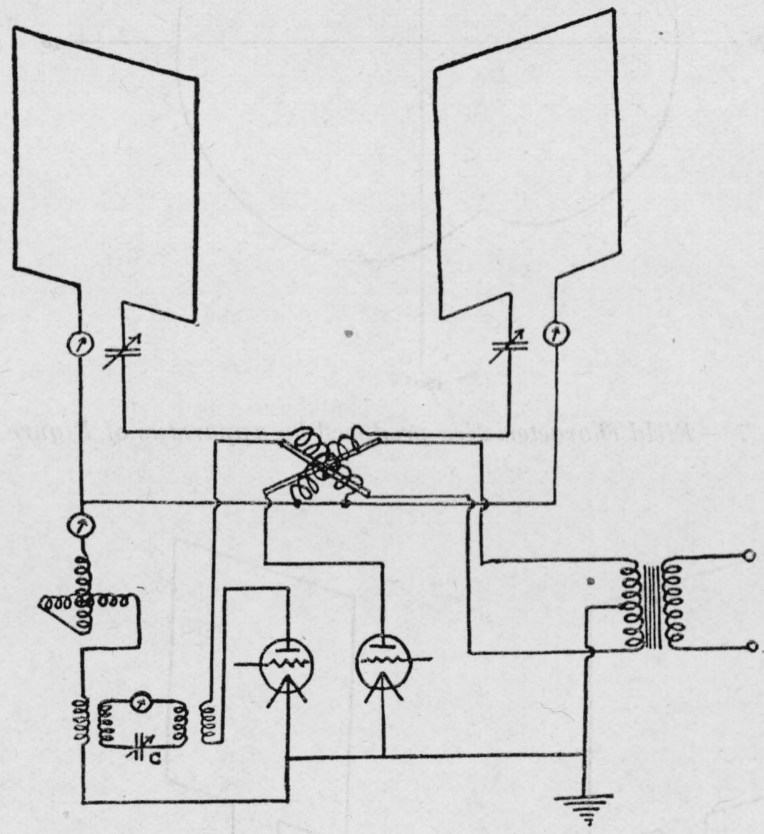

Fig. 6.-Use of loops as simultaneous radiators of directive and nondirective fields

The field resulting from this circuit arrangement is shown in Figure 7. This disappointing result led to an examination of the transfer of power to the variometer circuit. It was found that the majority of the power was being transferred capacitively through the transformer and that instead of the nondirective field being in phase with the directive field, it was close to $90^{\circ}$ ahead in time. Therefore, a delay circuit was interposed between the variometer circuit and the power source, as in Figure 8, to bring the two fields more nearly in phase. The improvement is seen in Figure $9(a)$, $(b),(c)$, and $(d)$, taken with progressively increasing nondirective fields. It is evident that none of these fields are sufficiently unidirectional for practical use. The back radiation from Figure $9(d)$, 


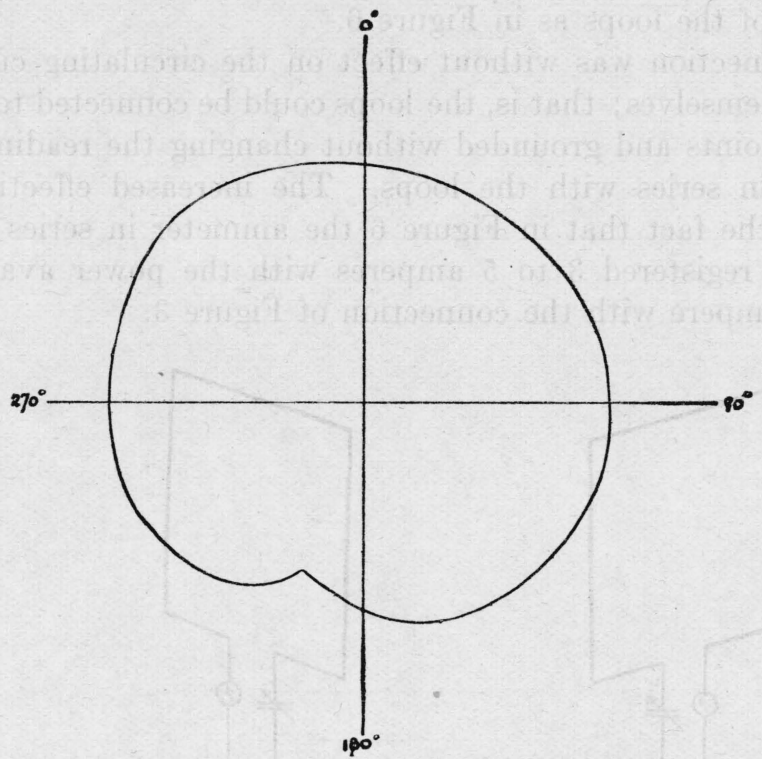

Fig. 7.-Field characteristics produced by apparatus of Figure 6

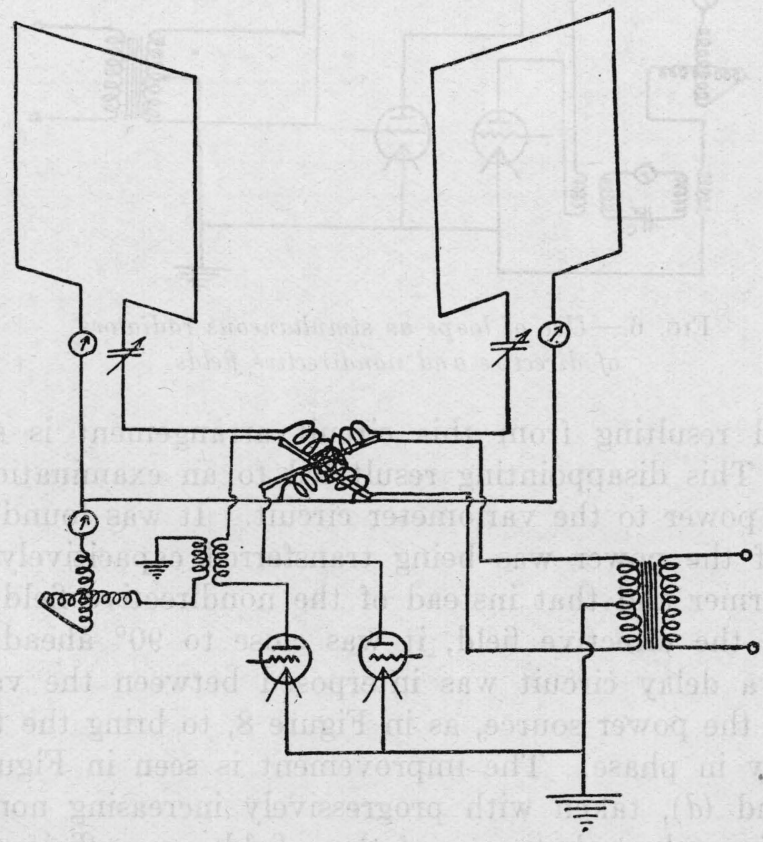

FIG. 8.-Same as Figure 6 with delay circuit 
for example, is still sufficient to give a powerful audible signal or to operate a sensitive indicator.

The circuit arrangement which finally gave the desired result is shown in Figure 10. The power for the nondirective field is obtained from an auxiliary goniometer whose primary windings are at $90^{\circ}$, and in series with the primaries of the goniometer controlling direction. Both goniometers were wound to minimize capacity transfer between windings. Hence, the phase of the nondirective field should be accurately controllable with the variometer, and a considerable

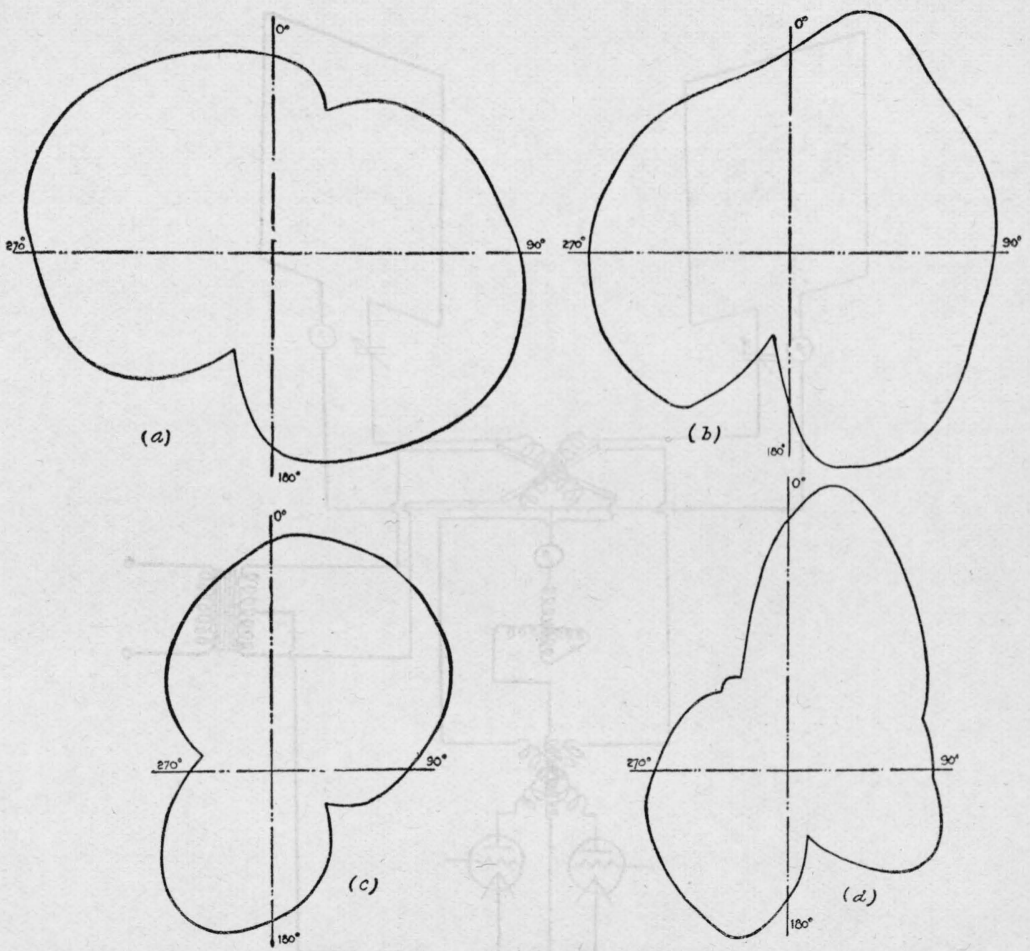

FIG. 9.-Field characteristics produced by apparatus of Figure 8

variation in amplitude is obtainable by change of the primary turns of the goniometer. By independent adjustment of these two, the appropriate phase and amplitude of the nondirective field with reference to the directive field, was found. A unidirectional field is obtained with the current $55^{\circ}$ different in phase one way or the other from the loop currents. Figure $11(a)$ shows the field with the nondirective field $55^{\circ}$ ahead of the directive field; Figure $11(b)$ with the nondirective field $55^{\circ}$ behind the directive field. With intermediate phase displacements, fields like Figure $11(c)$ are obtained. The fields of Figure $11(a)$ and $(b)$ can be rotated by means of the 
goniometer which normally controls the direction, leaving the remainder of the circuit unchanged during the rotation. This was proven by receiving the field at two locations, and checking the displacement of the maximum on the goniometer scale.

Since the marking out of courses is accomplished with modulations, such a polar field is useless unless the modulations retain their ability to form a sharp equisignal zone as explained in Section II. That they do is proved by directing the maximum of the polar field, say of Figure $11(a)$, at an observer, and adjusting the position of the sec-

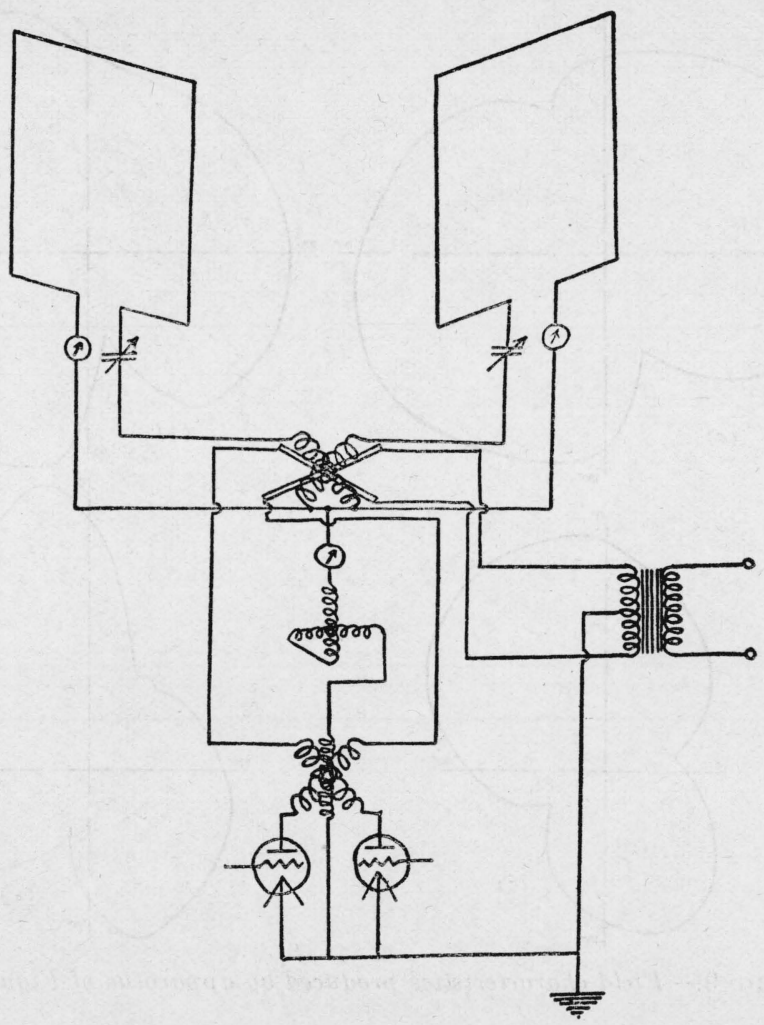

Fig. 10.-Final circuit arrangement

ondary of the auxiliary goniometer until the equisignal zone appears. This may be manifested audibly by the rise of an octave in pitch of the received note, or visually by proper operation of a course indicator. Under this condition, the most useful signal coincides in space with the maximum signal.

In Figure $11(a)$ and $(b)$, the arrows represent the angular position of the centers of the equisignal zones. It is seen that Figure $11(a)$ has but one radiated course instead of the usual four, and it occurs 
practically at the maximum of the field. Figure $11(b)$, while possessing an excellent polar characteristic, is not so acceptable from the standpoint of radiating useful signals. Two courses are radiated,
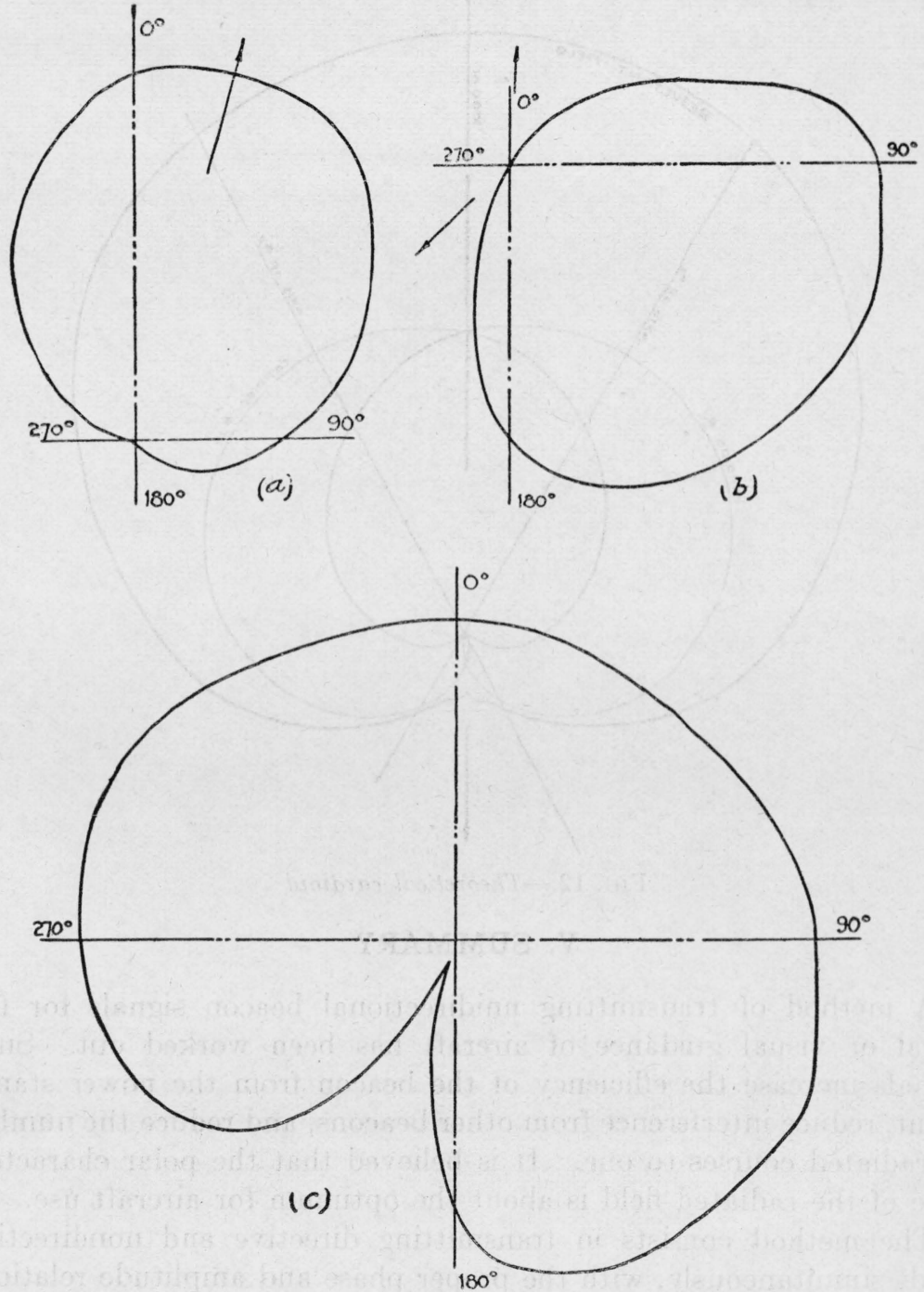

FIg. 11

$a$, Field at bureau, $L$ subtracted

$b$, Field at bureau, $L$ added

$c$, Field with wrong phase displacement

both very weak. Figure $11(c)$ is out of the question, both from the standpoint of the polar field and of the useful signal.

A "beam" for airplane reception must not be too narrow in order that the airplane may find it without too much difficulty. If it is 
too broad, the unidirectional effect is lost. It is believed that the fields of Figure $11(a)$ and $(b)$ represent about the optimum for airplane use.

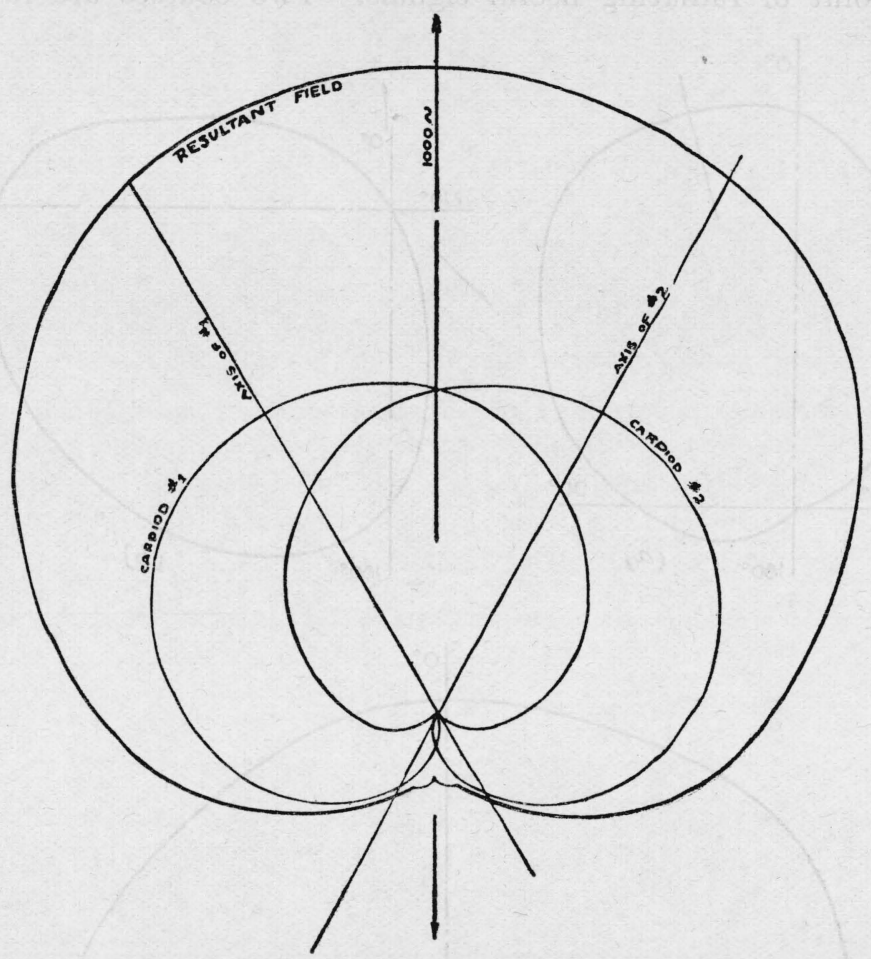

Fig. 12.-Theoretical cardioid

\section{SUMMARY}

A method of transmitting unidirectional beacon signals for the aural or visual guidance of aircraft has been worked out. Such signals increase the efficiency of the beacon from the power standpoint, reduce interference from other beacons, and reduce the number of radiated courses to one. It is believed that the polar characteristic of the radiated field is about the optimum for aircraft use.

The method consists in transmitting directive and nondirective fields simultaneously, with the proper phase and amplitude relations between them to secure unidirectional transmission.

Acknowledgments are due the American Telephone \& Telegraph Co. for the use of a private line connecting the beacon with two receiving locations in Washington; and to F. M. Baer, of the bureau's radio section, for valuable assistance in making the observations.

Washington, January 21, 1928. 